\title{
Trovatori catalani e unità aquitana
}

\section{Catalan Troubadours and Aquitaine unit}

RICCARDO VIEL riccardo.viel@uniba.it

Università di Bari Aldo Moro

Riassunto: Il saggio analizza i rapporti tra la produzione poetica dei trovatori aquitani e calatani in occitano del XII secolo, in particolare tra Guglielmo IX d'Aquitania, Marcoat e Marcabru. In questa prospettiva il mio saggio ha lo scopo di ricostruire il quadro delle relazioni storiche e linguistiche tra il mondo catalano, la Provenza e il Poitou, con una verifica sulle fonti manoscritte.

Parole chiave: trovatori catalani, Aquitania, letteratura medioevale

Abstract: The article will analyze the relationship existing between the lyric production of aquitaine and catalan Troubadours, with particular regard to Williams IX duke of Aquitaine, Marcoat and Marcabru. The study aims furthermore at reconstructing an outline of historical and linguistic relationships between Catalonia, Provence and Poitou with a survey of the manuscript sources.

Keywords: Catalan troubadours, Aquitaine, medieval literature

* Il presente articolo forma parte del PRIN 20179KMM4T_003.

DATA PRESENTACIÓ: 16/09/2020 ACCEPTACIÓ: 25/09/2020 • PUBLICACIÓ: 10/12/2020 


\section{Preambolo}

Per lungo tempo gli studî trobadorici hanno lasciato sedimentare l'idea che la lingua dei trovatori potesse essere descritta come una koiné sovraregionale, ossia come una lingua letteraria, di base provenzale, ma artatamente onnicomprensiva delle varie inflessioni dialettali dell'ampio dominio occitanico che, lo ricordo, comprende, oltre alla Provenza vera e propria ad est del Rodano, anche il Delfinato, l'Alvernia, la Linguadoca, il Limosino, il Perigordino, il Pittavino, la Guascogna e, secondo alcuni linguisti, anche il sistema catalano. I trovatori avrebbero dunque, volta per volta, scelto d'innervare, su una base fondamentalmente provenzale, tratti talora limosini, talora alverniati, talaltra linguadociani, e via dicendo, a seconda delle esigenze di rima o di prosodia.

Tale impostazione ha spesso lasciato cadere, nelle singole edizioni critiche, lo studio linguistico dei trovatori, perché si riteneva vano dare importanza geolinguistica ai tratti regionali rintracciabili nella lingua dei singoli poeti. Una prospettiva, questa, che non tiene conto dell'ampia campitura cronologica del fenomeno trobadorico, che si estende per quasi due secoli di produzione letteraria, circolazione di testi, trascrizione e infine ricezione. Un fenomeno troppo lungo per poter pensare che sia nato con una koiné artatamente studiata e calibrata, mantenutasi inalterata e intatta sia nel suo ruolo letterario, sia nel suo ruolo politico-culturale e sociale.

Del resto la gran parte della filologia provenzale si è esercitata, dai primordî sino ad oggi, nella prassi dell'edizione di corpus, ossia sull'edizione del canzoniere di un singolo poeta, che è poi difficile inquadrare nell'ampio panorama linguistico del movimento trobadorico. Oppure, all'inverso, ci si è molto dedicati all'analisi dell'intera tradizione manoscritta della lirica trobadorica senza soffermarsi sullo studio del singolo - o dei singoli - trovatori. Ancora, sono stati compiuti ottimi lavori di scavo dei singoli canzonieri, ricercandone la stratigrafia linguistica e la sedimentazione delle fonti, senza però riuscire a valutare con questo la lingua dei primi e più antichi trovatori.

La grande scuola storica di fine Ottocento aveva tutt'altra impostazione, se è vero come è vero che un grande filologo come Gröber aveva inteso studiare i rapporti tra i canzonieri provenzali al fine di aiutare il filologo nell'analisi filologica dei testi dei singoli trovatori, e se è vero come è vero che in questo periodo Brunel studiava la scripta occitanica dei documenti con i suoi studî su Les plus anciennes chartes en langue provançal con l'intento di aiutare l'analisi della scripta letteraria dei singoli trovatori.

\section{Tracce di una centralità della scripta aquitana e trobadorismo catalano}

Sappiamo bene che il primo trovatore noto è Guglielmo IX, conte di Poitiers e duca d'Aquitania, vissuto a cavallo tra l'XI e il XII secolo. La prima raccolta scritta di testi provenzali a noi giunta, fatte salve alcune testimonianze indirette nel Jaufre de Poitiers, è il canzoniere provenzale estense siglato dai provenzalisti D, esemplato in pianura Padana nel 1254, ossia centocinquant'anni dopo Guglielmo IX. Di quel mezzo secolo di produzione letteraria noi possiamo solo ipotizzare forme 
di tradizione scrittoria di cui sappiamo ricostruire desultoriamente alcuni passaggi, come ipotetiche raccolte autoriali, libercoli di giullari, fonti più o meno organizzate, direi meno che più. Appare inverosimile ritenere che la scripta tramandata dai canzonieri trascritti dai centocinquanta a duecento anni dopo la nascita del trobadorismo corrispondesse alla scripta originale che dovevano aver adottato i più antichi trovatori.

Per provare, ad ogni modo, che vi sia stata un'evoluzione nella scripta trobadorica, è importante ricostruirne le discontinuità; e una discontinuità mi pare di poter intravedere nella nascita del trobadorismo catalano.

Come si sa, i rapporti tra la Catalogna e la Provenza si saldano con l'unificazione tra la contea di Barcellona e la contea di Provenza sotto Raimondo Berengario III il Grande a partire dal 1113. Tuttavia l'unità catalano-provenzale rimane assoggettata al potere feudale di Alfonso VII di Castiglia almeno per tutta la prima metà del XII secolo. Sarà solo con le nozze tra Petronilla, figlia del re d'Aragona Ramiro II il Monaco, e Raimondo Berengario IV di Barcellona, che si avrà un accrescimento non solo dell'importanza dell'asse catalano-provenzale, ormai acquistando il regno d'Aragona, ma anche una sua sempre maggiore indipendenza dalla corona di Castiglia.

Prima di tutto ciò, tuttavia, uno dei centri più importanti del Medioevo centrale era il regno e poi ducato d'Aquitania. L'indipendenza di fatto del ducato d'Aquitania, sebbene formalmente vassallo alla corona francese, determinò la nascita di una corte potente e colta, almeno a partire da Guglielmo IV Braccio di Ferro e del successore Guglielmo il Grande. Dal 1058, poi, con Guglielmo VIII, l’Aquitania annesse a sé anche la Guascogna.

Il passaggio dal predominio politico e culturale del ducato aquitano alla saldatura e alla crescita politica dell'influenza dell'asse catalano-provenzale è dunque un dato di discontinuità storica molto evidente, che si consuma a cavallo tra il XII e il XIII secolo.

A questa discontinuità storica corrisponde una discontinuità culturale. Guglielmo IX è l'erede della ricca e florida corte aquitana. I rapporti tra l'area pittavina e la Penisola iberica sono noti, e molto probabilmente dipendono, anche in questo caso, dall'antica unità aquitana che rappresenta, fino alla costituzione dell'unità francese sotto Filippo Augusto, il principale fulcro culturale dell'area galloromanza. Si tratta di un asse Nord-Sud che collega l'area transpirenaica alla Francia meridionale. Con la dinastia plantageneta questo asse è destinato a un ruolo ancor più importante, giacché metterà in comunicazione l'area trans-pirenaica con la Francia del Nord e le isole britanniche.

A Guglielmo IX seguono alcuni trovatori di aree limitrofe, come Jaufre Rudel, Cercamon, e Marcabru. Solo pochi sinora si sono interrogati su quale scripta Guglielmo IX e i trovatori gravitanti attorno quella corte avessero adottato (fondamentali restano i saggi di Pfister 1976 e Jensen 1986). La risposta più ovvia è che essi dovessero in qualche modo appoggiarsi alla scripta della corte aquitana, il cui centro culturale era Poitiers. In questo senso ho già mostrato altrove come nei trovatori più antichi gravitanti attorno Guglielmo IX e Guglielmo X, e soprattutto in Marcabru, 
un'accorta rivalutazione della varia lectio di alcuni manoscritti e di alcune fonti, tra le quali un'antica fonte che ho chiamato $v$, ma anche la fonte $\omega$, rimettesse in luce hapax e vocaboli rari (Viel 2017). Quasi sempre tali vocaboli recuperati possono essere interpretati come guasconismi, oppure come oitanismi o più probabilmente pittavinismi. Dunque Guglielmo IX, i trovatori gravitanti attorno alla sua corte, e soprattutto Marcabru, adottavano nella loro lingua una commistione di elementi pittavini, oitanici e guasconi (Viel 2015). Richiamo qui brevemente i tratti più significativi, utili a dare misura del fenomeno. Innanzi tutto ricordavo, nel mio saggio, gli esiti pirenaici della prima persona singolare del perfetto in -ic (Rohlfs 1970: 216), in Lo vers comenssa:

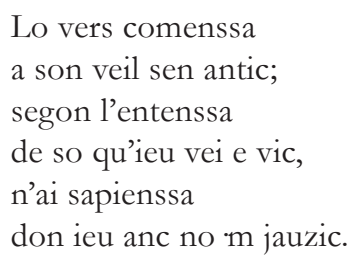

Vi è poi l'interpretazione del bacucs di $A l$ departir del bran tempier come continuatore del guascone bagàn 'ozioso' attraverso il basco bakant 'raro' (Barachini-Viel 2016: 45-46):

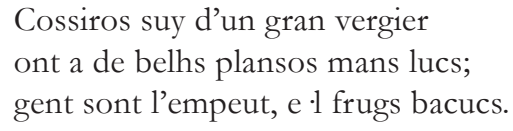

Il passo sarebbe dunque da tradurre, accettando e integrando l'interpretazione di Roncaglia 1953: «Ho nella mente un gran giardino ove si trovano più boschetti di belle piante; nobili sono gli innesti, ma il frutto è vacuo / inconsistente / inutile / ozioso». Si ha infine il verbo cogular di L'autrier a l'issuda d'abriu, che può essere ricondotto all'antico guascone acoucoulà nel senso di 'accovacciarsi' / 'essere affettuosi' (FEW IV 160b e Palay 1961: 11):

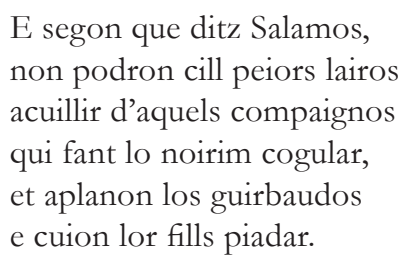

In traduzione: «E secondo quanto dice Salomone, costoro non possono accogliere i ladri peggio di coloro che fanno accovacciare la nidiata, e i mariti che accarezzano piccoli bastardi e pensano di essere affettuosi con i loro figli». Infine vi è la probabile rivalutazione della variante res fanissa del ms. a in L'autrier jost'una sebissa (v. 9) nel senso di 'creatura di grazioso portamento' (Meneghetti 1993: 


\section{Riccardo Viel. Trovatori catalani e unità aquitana}

192-193), riconducendola all'a.cast. alfeñique, a.val. alfaní, port. alfenim, tutti dall'ar. fanîq 'molliter habita atque educata (puella)'.

Tuttavia accanto a tali esiti sud-occidentali se non propriamente guasconi o iberici coesistevano esiti oitanici, come la ripetuta presenza in rima di esiti dell'evoluzione $-e i \mathrm{da}-e$ chiuso finale in mercey, crey, tey, rey, la così detta «rima pittavina», riscontrabile in numerosi vers di Marcabru: $A$ la fontana del vergier, Emperaire, per vostre prez, En abriu, Estornel, cueill ta volada. Molto forte è la traccia di fonetica oitanica nella parola meire col significato di 'medico', in El son desviat chantaire:

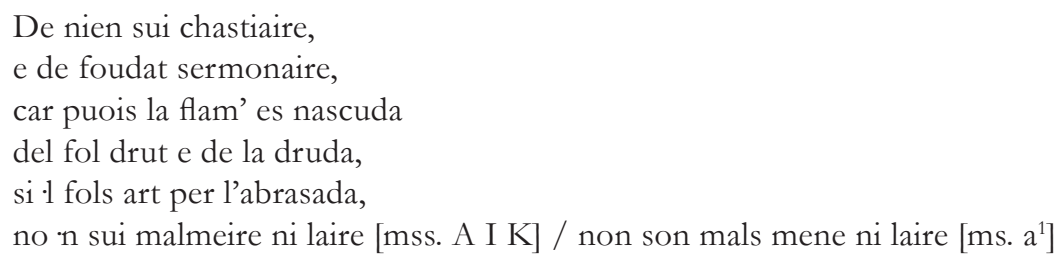

Avevo già suggerito nel saggio citato di ricondurre la forma, anziché a un mal meire 'blâmable' (Dejeanne 1909), o a un mal merens, da merir, 'not guilty' (Gaunt-Harvey-Paterson 2000), all'afr. mire, meire 'medico', traducendo il passo: «Invano sono censore e sermonatore della follia, perché dopo che la fiamma è sorta dagli amanti folli, se il folle arde per la fiamma bruciante, non sono né un cattivo guaritore né un ladro» (Viel 2015: 11).

Si veda ancora il fortissimo chen 'cane' (< lat. CANIS) in rima al v. 56 di Pois l'inverns d'ogan es anat\%, con palatalizzazione della vocale tonica (Perugi 2003: 575).

Anche a livello della scripta si può notare un'analoga commistione. Accanto a fenomeni di stampo guascone, è probabile ipotizzare l'adesione di Marcabru ad alcune norme scriptologiche aquitane. Questo lo si nota in alcune rime, che ci appaiono oggi imperfette, e che rispondono probabilmente alle norme dell'assonanza della poesia mediolatina di [o] chiusa con [u] e di [e] chiusa con [i], ben testimoniate dai primi testi romanzi pittavini come la Passion di Clermont-Ferrand studiata da D’Arco Silvio Avalle (Avalle 1962: 88-91).

In Marcabru una possibile rima tra [o] chiusa e [u] potrebbe trovarsi in A l'alena del vent doussa, anche se il componimento è stato oggetto di differenti e complessi interventi ricostruttivi miranti proprio a ridurre la presunta irregolarità rimica. La restituzione migliore dello schema rimico, che ricorre a interventi ecdoticamente meno numerosi oltreché meno onerosi, è quella a cui si giunge in Lazzerini 1990 e Beltrami-Vatteroni 1988-94: II, 241, simile a Ricketts 1986, dove si ritiene accettabile la rima on: om: $\mathrm{um}^{1}$. Ancora più stringente l'inevitabilità della rima tra le due vocali velari

1 Personalmente ritengo di poter proporre, accettando l'unico intervento di ritocco testuale sul rimante del v. 22 in vau cobert om suggerito in Lazzerini 1990, lo schema rimico di Ricketts 1968 letto come fosse una strofe formata da una fronte di un verso a rima variabile $\mathrm{a} / \mathrm{b}$, una sirma di due volte, la prima composta da tre versi a rima variabile $\mathrm{a} / \mathrm{b}$,

SCRIPTA, Revista internacional de literatura i cultura medieval i moderna, núm. 16 / desembre 2020 / pp. 416-430 ISSN: 2340-4841 $\cdot$ doi:10.7203/SCRIPTA.16.241 
in Al prim comenz de l'ivernaill, nella ricostruzione di Lazzerini 1992, che ipotizza una serie rimica sanglot: salutr: agutæ: ecc. Per la rima tra le vocali palatali, ossia [e] chiusa con [i], si potrebbe citare il probabile caso della serie rimica di Pois l'iverns d'ogan es anatz, dove alla cobla IV si ha un mantener in rima -ir (a meno che non si debba qui ipotizzare un oitanismo originario dall'afr. maintenir).

Per quanto riguarda i testi delle origini, si possono innanzi tutto citare alcune lasse della Passion:

\author{
Lassa Xxxv \\ Eu soi aquel!» zo dis Jesús. \\ Tuit li felun cadegrent ios. \\ Terce vez lor o demanded, \\ a totas treis chedent envers. \\ Lassa cXxviII \\ Christus Jesús, qui mans ensus, \\ mercet aias de pechedors; \\ en tals raizons si a mmaespraes, \\ per ta pitad lo $\mathrm{m}$ perdones. \\ Lassa XXIV \\ Et per lo pan et per lo vin \\ fort saccrament lor commandez, \\ per remembrar sa passiun, \\ que faire rova a trestot; \\ Lassa XuIX \\ Anz quae la noit lo jalz cantes, \\ terce vez Petre lo neiez. \\ Jesús li bons lo rewardet, \\ lui recognostre 1 semper fiz.
}

Si possono citare anche lasse dallo Sponsus (1965) e dal Boeci (1963):

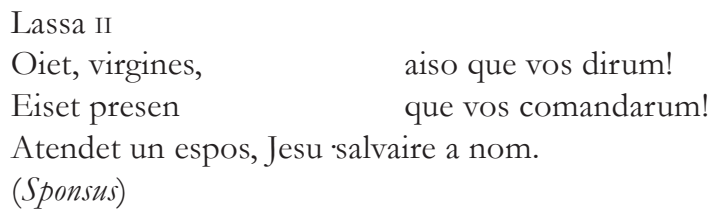

la seconda rappresentata dalla rima fissa $\mathrm{x}$. Si otterrebbe così lo schema assolutamente regolare, che giustificherebbe strutturalmente anche la tornada, che sinora era stata sempre ritenuta irregolare e irriducibile: strofe 1: a-bbbx; strofe 2: b-bbax; strofe 3: b-aabx; strofe 4: a-bbbx; strofe 5: b-bbax; strofe 6: b-aabx; strofe 7: a-bbbx; tornada: bbax. In questo modo si avrebbero due permutazioni, dove la fronte e prima volta della sirma compiono due cicli: a-b-b, a-b-b la fronte, bbb-bba-aab, bbb-bba-aab la prima volta, e poi ripetono l'ordine originario nella settima strofe: a bbb. La tornada ripete la sirma nella combinazione immediatamente successiva quella della prima strofe: bbax. Si tratterebbe di una sorta di retrogradatio, con una rima fissa. 


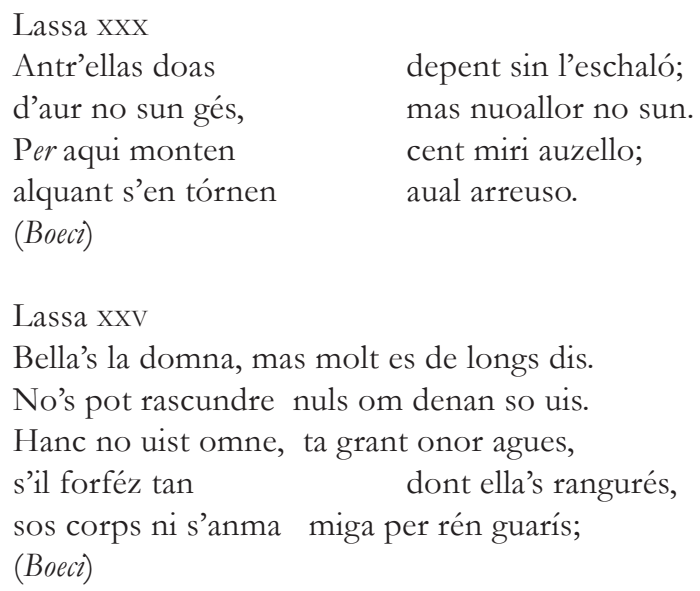

La mia tesi è che i trovatori più antichi scrivessero in una scripta ancora vicina alle abitudini mediolatine della curia aquitana, e che adottassero un pittavino aulico misto a fenomeni guasconi e pirenaici. Di tale patina linguistica è andato perso quasi tutto, se non rare tracce che ancora emergono nel corpus poetico di Marcabru.

In questo torno d'anni esistevano già trovatori catalani: ricordo infatti che lo stesso Marcabru aveva operato al di qua dei Pirenei, presso la corte castigliana di Alfonso VII. I più antichi trovatori genuinamente catalani sono, probabilmente, Alegret e Marcoat. A quest'epoca l'influsso culturale della corte aquitana era ancora predominante, e infatti sembra che anche questi trovatori avessero adottato una scripta aquitana simile a quella di Marcabru, di cui erano sodali.

Alegret era probabilmente un non ignoto Alegret di Claverol, attestato nei documenti quale esponente di una famiglia di piccola nobiltà catalana, vassallo dei conti di Pallars-Sobirà e di PallarsJussà (Viel 2014: 285-288). I suoi testi presentano indizî linguistici prevalentemente lessicali, ad esempio mec nel significato di 'bègue', al v. 6 di Ara pareisson ll'aubre sec, guasconismo e catalanismo semantico (Viel 2011: 35). Il trovatore presenta anche alcuni tratti sintattici, come l'oggetto diretto espresso con $a+$ accusativo (Rohfls 1970: \496), reperibile nella seconda cobla di [Ai]ssi cum selh, dove si legge:

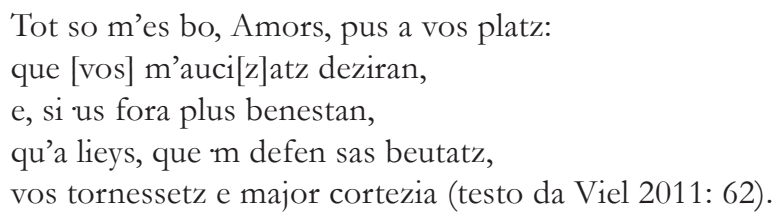


Il passo è dunque da tradurre: «Tutto ciò mi piace, Amore, giacché a voi piace: che voi m’uccidiate desideroso e, se vi fosse più gradito, che mutaste in maggior cortesia lei, che mi nega le sue beltà».

Di Marcoat abbiamo, invece, catalanismi più evidenti. L'analisi dei tratti fonetici in rima ci restituisce un'immagine linguistica omogenea e coerente. Si legga il seguente passo (Una ren os dirai En Serra, vv. 25-27):

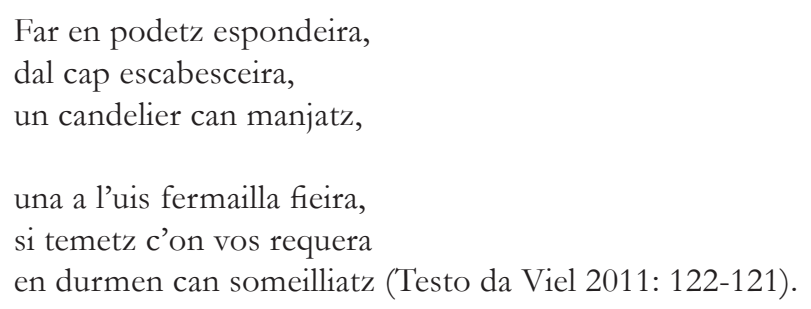

L'apparizione della forma fieira, al posto di fivela, in rima, dal lat. FIBULELLA, rappresenta un fortissimo guasconismo fonetico. Si tratta infatti dell'evoluzione della -LL- intervocalica in $/ \mathrm{r} /$, che è una ineludibile spia di derivazione guascona e pirenaica (Rohlfs 1970: \$S 468, 518; LRL II/2: 452, 457). Emerge un guasconismo e catalanismo molto forte anche nel sistema di rime plana demana e destena contena arena lena ecc., dove si notano le forme demana e contena al posto di demanda, destenda e contenda: l'assimilazione del gruppo originario -ND- è un tratto caratteristico del guascone, della zona pirenaica e del catalano (LRL II/2 : 452, 457 ; Ronjat 1932 : \339; Rohlfs 1970 : \471).

Infine l'utilizzo del dimostrativo latino IPSE, IPSA nei primi versi di Marcoat come articolo determinativo non lascia adito a dubbî circa l'origine catalano-pirenaica del brano (LRL II/2 : 499, \5.4.1.3., 460; Rohlfs 1970: \490; Grafström 1968: 29). Da ultimo si noti che la prima attestazione del lemma picarel si trova in Marcoat; in séguito, a partire dal XIV-XV secolo, il termine si ritrova solo nell'area iberica.

Ma vi sono anche dei dati che legano Marcoat e Alegret all'area pittavina. Innanzi tutto il tipo metrico dei sirventesi di Marcoat, il versus tripertitus caudatus, che era utilizzato soprattutto nell'innologia mediolatina di San Marziale di Limoges. In secondo luogo la rima farina con arena, Serena, destena, lena, escofena ecc. nel sirventese di Marcoat Mentre m’obri (vv. 28-30):

\footnotetext{
Almornes e morsels crus assunaras al temps brus, e sal e meill e tfarinat.
}

Nell'edizione critica da me curata mi ero arreso a segnalare l'infrazione rimica con una crux interpretum accanto al lemma farina, suggerendo in nota la congettura ope ingenii «codena», scrivendo: «Dans la cobla conclusive on voit cités de la nourriture et des ingrédients utiles pour la subsistance 
hivernale (...). Un autre alimen utilisé pour la conservation de la viande au Moyen Âge (...) était la couenne, que l'on utilisait tantôt pour faire des soupes tantôt dans la préparation des charcuteries (...). La genèse de la faute d'archétype s'explique probablement par l'influence logique de "meill" qui, par association, a donné "farina" plutôt que "codena"» (Viel 2011: 117). Ora posso invece accettare la lezione dei manoscritti «farina», e ricondurre questa presunta irregolarità alla assonanza tra [e] chiusa e [i], perfettamente ammessa nella poesia mediolatina, attestata secondo Avalle nella Passion di Clermont-Ferrand.

Insomma, i due più antichi trovatori catalani, fioriti nella prima metà del XII secolo, probabilmente adottavano una lingua ancora vicina alla scripta aquitana, con elementi di commistione linguistica simili a quelli ancora visibili nei testi di Marcabru e Guglielmo IX: elementi fonetico-lessicali pittavini, guasconi, catalani, ed elementi fonetici e metrici derivanti dalla poesia sacra e profana mediolatina.

\section{Trovatori catalani e contea di Provenza}

Una vera e propria scuola di trovatori catalani nascerà solo nel secondo terzo del XII secolo, con Guillem de Cabestaign, Guiraut de Cabreira, Guillem de Berguedan, Pons de la Guarda, Peire Gauseran, poeti tutti più o meno legati al monarca Alfonso II, egli stesso trovatore. Nei testi che ci sono tramandati, la loro lingua appare come un occitanico ben legato al provenzale in senso stretto, anche se venato di alcuni catalanismi lessicali e morfologici, ben individuati da Martín de Riquer per Guilliem de Berguedan, da Cots e prima già da Långfors per Guilhem de Cabestanh. Si può trattare di un articolo determinativo derivante dal lat. IPSE, di alcuni catalanismi semantici, di spie lessicali anche più nette. Solo in pochi casi, e in trovatori meno solidamente gravitanti all'interno della corte alfonsina, come Guillem de Cabestaing, possono rinvenirsi casi di catalanismi fonetici o di scripta in rima, come rime tra [e] aperta ed [e] chiusa. In generale, però, la loro lingua è ben saldamente provenzalizzata, e non si trovano più relitti fonetici, lessicali o morfologici imputabili alle varianti settentrionali dell'area occitanica, come il pittavino o il limosino, affioranti in Alegret e Marcoat e nei poeti della generazione guglielmina e marcabruniana.

Si può dunque vedere nell'accresciuta indipendenza del dominio alfonsino, esteso dall'Aragona, alla contea di Barcellona, alla contea di Provenza, il momento di fondazione del trobadorismo catalano. In tal senso è anche possibile riflettere circa la lingua che tale «scuola» di trovatori doveva aver sussunto, perché credo che sia proprio questo il momento in cui l'aumentata influenza politica dell'asse catalano-provenzale si fece elemento trainante di una nuova scripta e di un nuovo polo culturale e linguistico allinterno del trobadorismo.

È nota la rivalità tra il re Casto e il marchesato di Provenza, e con figure quali Raimondo di Tolosa, alla cui sorte politica è legato il noto poeta Raimbaut d'Aurenga, e le corti del tolosano. Fu con Raimondo Berengario IV, conte di Provenza dal 1209, erede di Alfonso II d'Aragona, che la corte di Provenza divenne un vero e proprio centro attorno cui gravitavano molti trovatori. Dal 
punto di vista poetico e letterario le varietà linguistiche in gioco erano ormai quelle della Provenza propriamente detta (contea e marchesato di Provenza), dove rivaleggiano la corte di Raimondo Berengario, con poeti del calibro di Elias de Barjols, e quelle del Baux.

\section{Il provenzale delle Razos de trobar}

Insomma, mi appare sempre più chiaro che la scuola catalana livelli, intenzionalmente, la propria scripta poetica sul modello provenzale propriamente detto, quello cioè della Provenza ad est del Rodano, e voglia promuovere questa nuova scripta all'intera cultura trobadorica.

Di tutto ciò la testimonianza più evidente è l'opera di Raimon Vidal de Besalú; e non faccio qui riferimento alla sua opera di trovatore, ampiamente studiata da Giuseppe Tavani, quanto a quella di grammatico. Nel 1210, è noto, Raimon Vidal compone, alla corte catalana di Hug de Mataplana, le Razos de trobar, vero e proprio trattato retorico, poetico e linguistico dell'arte del trobar. Si tratta della più antica grammatica di provenzale mai scritta, e non a caso esemplata in area catalana.

Le correzioni di Raimon Vidal vertono su alcuni tratti significativi di fonetica, di cui il primo che vorrei prendere in esame è il seguente:

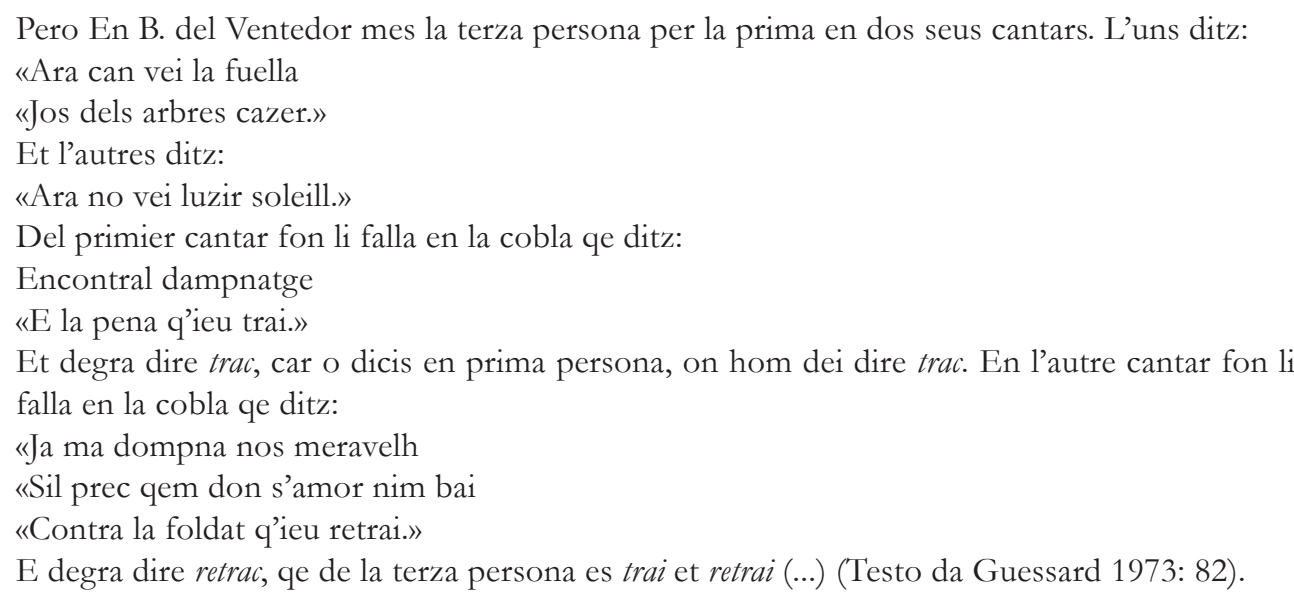

Si tratta in questo caso del trattamento della consonante occlusiva velare intervocalica. Raimon Vidal individua la forma trai dal medio latino TRAGO, presente indicativo, correggendolo in trac. In effetti l'evoluzione in vocale /i/ della velare è un tratto tipico dell'area settentrionale del dominio occitanico, in particolare limosino e perigordino, e buona parte del pittavino (LRL, II/2: 416; Pignon 1960: 429 ss., in part. 434, soprattutto per -C- + A). Fenomeno consimile è aspramente criticato da Raimon nelle forme amis per amics, dove la caduta della -c finale è propria del francese: si tratta di un tratto oitanico; in pittavino /-c/ si mantiene (Pignon 1960: 474-476). 


\title{
Riccardo Viel. Trovatori catalani e unità aquitana
}

Si veda infatti questo brano delle Raz̧os:

Poi vos dic qe tuit cill qe dizon: amis per amics, et mei per me an fallit, et mantenir per mantener, et retenir per retener, tut fallon, qe paraulas son Franzezas (Guessard 1973: 85).

In questo passo ancor più evidente è la posizione anti-pittavina e anti-aquitana, giacché Raimon stigmatizza la forma mei per me. Si tratta dell'evoluzione di E lunga latina in /ei/, tipica dell'area pittavina e molto diffusa in Gulglielmo IX e Marcabru (LRL, II/2: 369). Nello stesso luogo Raimon critica le forme mantenir, contenir, retenir, che sono metaplasmi di coniugazione, suggeriti dalle forme oitaniche, per gli occitanici mantener contener, retener. Anche in questo caso tali forme sono diffuse nei trovatori più antichi di area nord-occidentale.

Consideriamo un terzo passo delle Raz̧os:

\begin{abstract}
Per aver mais d'entendemen vos vuoil dir qe paraulas, i a don hom pot far doas rimas aisi con: leal, talen, vilan chanson, fin. Et pot hom ben dir, qi si vol: liau, talan, vila, chanso, fi. Aisi trobam qe o an menat li trobador; mas li primier, so es leal, talen, chanson, son li plus dreig. Vilan, fin, suffren miels abreviamen (Guessard 1973: 85).
\end{abstract}

In questo passo, pur ammettendo la possibilità di scelta tra le varianti fonetiche, Raimon Vidal consiglia ancora una volta gli esiti proprî dell'area tipicamente provenzale e linguadociana, ossia centro-meridionale, rigettando gli esiti settentrionali e specialmente pittavino-limosini. Ad esempio, consiglia l'uso di leal anziché liau, dove si nota la vocalizzazione della laterale finale, fenomeno tipico del pittavino, limosino, perigordino, e del guascone, tardivamente attestato nel delfinatese: si tratta di un tratto caratteristico dell'unità aquitana (LRL, II/2: 371 per il limosino e saintongese, p. 417 per il limosino e perigordino, p. 457 per il guascone; per il pittavino si veda anche Pignon 1960: 465-469; per il delfinatese Bouvier 1976, sez. 4, cap. 2). Ancora, la forma talan dal latino TALENTUS, è vista come da evitare rispetto a talen, con conservazione del timbro della vocale tonica; questo perché nella prima forma si riscontra una nasalizzazione del nesso /en/, con oscillazione /an/ / en/ tipica dell'area oitanica e diffusa nell'area pittavina e nel settentrione del dominio occitanico (LRL, II/2: 415, \3.2.2.). Nel consigliare le forme chanson, vilan e fin si nota anche una certa avversione al fenomeno della caduta della /n/ finale, che si riscontra soprattutto, ancora una volta, nel limosino, perigordino, alvergnate centro-settentrionale, delfinatese alpino solo per le vocali chiuse, ma in questo caso anche nel Linguadociano (ma non in Guascogna, né in pittavino), dunque in tutta l'area occitanica centrale a ovest del Rodano (per il limosino e perigordino: LRL, II/2: 416 \3.3.4.; per l'alvergnate: LRL, II/2: 424; per il delfinatese: LRL, II/2: 439, \ 3.2.7, per il linguadociano: LRL, II/2: 446, \3.2.4.). 


\section{Alcune prime considerazioni conclusive}

Si deve dunque osservare che la più antica grammatica provenzale nasce all'alba del XIII secolo, e in ambiente catalano. Mi sembra di aver dimostrato che l'intento di Raimon fosse quello di stabilire una koiné poetica, che consiste quasi nella depurazione della lingua dei trovatori degli elementi aquitano-pittavini e settentrionali, preservando forme, esiti fonetici e morfologici tipicamente provenzali, specificamente derivanti dall'area a est del Rodano. Si può dire che l'operazione di Raimon sia quella di mettere al centro della letteratura trobadorica la contea di Provenza.

A questo punto è però evidente che una presa di posizione così netta nelle Razos de trobar deve configurarsi come una «reazione» forte a una scripta preesistente. Non sembra, infatti, determinata dalla ragione di comprendersi: la comprensione reciproca tra le lingue pittavina, guascona, provenzale e catalana non sembra essere mai stata messa in dubbio; neppure tra le varianti oitaniche e quelle più strettamente occitaniche. Mi pare, invece, che tale reazione vidaliana nasconda il tentativo, ormai maturo e per questo riuscito, di sostituire all'antica scripta aquitana, mista di elementi guasconi, pirenaici e pittavini, una nuova scripta prettamente provenzale, trans-rodaniana. Un'operazione linguistica che è assieme operazione culturale e operazione politica. L'asse catalano-provenzale, l'unione tra la contea di Barcellona, il regno aragonese e la contea di Provenza si sostituisce al tramontante asse aquitano, sconfitto dal tramonto dei Plantageneti e dal distacco tra la contea di Poitiers e le corti del midi occitano. È in questo torno d'anni, in questa operazione politico-culturale, che matura e fiorisce il trobadorismo provenzale-catalano, e si pone come orizzonte vincente sino all'inizio del XIV secolo.

Ecco dunque che Raimon Vidal mette nero su bianco un'idea di lingua trobadorica pura, che tende a stigmatizzare tutti i tratti a ovest e a nord del Rodano. Di fatto vengono progressivamente escluse tutte le possibili varietà fonetiche o morfologiche riconducibili all'alverniate, al perigordino, al limosino, al pittavino e financo al linguadociano. Il tratto più interessante di questa rifondazione della scripta trobadorica è che lo stesso Raimon ritiene opportuno chiamare questa lingua, rigidamente provenzale, col nome di lemosí, ossia limosino:

Totz hom qe vol trobar ni entendre dei primierament saber qe neguna parladura no es tant naturals ni tant drecha del nostre lingage con aqella de Proenza o de Lemosi o de Saintonge o d'Alvernga o de Caerci. Per qe ieu vos dic qe qant ieu palarai de Lemosis, qe totas estas terras entendas, et totas lor vezinas, et totas cellas qe son entre ellas. Et tot l'ome qe en aqellas son nat ni norit an la parladura natural et drecha; mas cant us de lor es issitz de la parladura per una rima o per alcun mot qe li sera mestier, cuion las genz qi non entendon qe la lur lenga sia aitals, qar non sabon lur lenga; per qe mielz lo conois cel qi ha la parladura reconoguda que sel qi non la sap, e per zo non cuion mal far qan geton la parladura de sua natura, anz cuion qe sia aitals la lenga. Per q'ieu vueil far aquest libre per far reconoisser las parladuras d'aquels qi la parlon drecha, e per enseignar aicels qui non la sabon (Guessard 1973: 71).

In realtà questa lingua di limosino, come lo intendiamo noi oggi, ossia come varietà settentrionale dell'occitano parlata nella regione di Limoges, non ha nulla. La lingua di «qi la parlon drecha», come 
dice Raimon, non è altro che la lingua dei nativi della Provenza, ad est del Rodano, nonostante i più antichi trovatori, come Guglielmo IX, Marcabru, Bernart de Ventadorn, Giraut de Bornelh, fossero limosini, pittavini, saintongesi. Ma proprio in questo sta la cifra della forte reazione, a mio parere, del trobadorismo catalano-provenzale, alla lingua dei trovatori antichi: sta nell'appropriazione dell'etichetta stessa della lingua. Limosino è per antonomasia il nome della lingua dei trovatori, proprio perché i trovatori più antichi, Guglielmo IX, Marcabru, Jaufre Rudel, Giraut de Borneil, Bernart de Ventadorn, usavano una scripta occitanica propbabilmente più prossima al limosino-perdigordinopittavino che al provenzale. E Raimon Vidal si appropria di questa etichetta, riempiendola di un nuovo contenuto grammaticale e normativo. Si potrebbe quasi dire, per esemplificare, che Raimon Vidal rigetti, del limosino, tutto ciò che è limosino, imponendogli tutto ciò che è provenzale.

Questa imponente frattura, questo passaggio da una scripta occitano-settentrionale a una scripta occitano-provenzale, è testimoniata dunque dalla nascita e dallo sviluppo della scuola dei trovatori catalano-provenzali, attorno alla corte del conte di Barcellona, della contea di Provenza, della corte dei Baux, di Beatrice di Savoia signora di Aix, dei Reforsat de Trets signori di Marsiglia, dei Blacatz signori di Aups, e di tutto il territorio implicato. Ciò testimonia altresì un’importante cesura tra i trovatori delle prime generazioni e quelli della generazione del così detto manierismo trobadorico, che rappresenta una gran parte della produzione lirica del XIII secolo (Folchetto da Marsiglia, Elias de Barjols, Blacatz, Blacasset, Falquet de Romans, ecc.).

Cosa accade nei canzonieri provenzali? Come accennavo, essi sono esemplati a partire dalla seconda metà del XIII secolo, dunque dopo questa rifondazione normativa professata nelle Razos de trobar di Raimon Vidal e in altre grammatiche coeve, come il Donatz proenzals. I canzonieri vengono esemplati in maggioranza nel Nord Italia e poi, a partire dall'inizio del XIV secolo, anche in Linguadoca e nella Penisola Iberica. Chiaramente i tratti in rima, sui quali si concentra maggiormente l'acribia correttoria di Raimon Vidal, rimangono intatti, perché difficilmente il copista interveniva modificando la fonetica dei rimanti, altrimenti avrebbe causato delle infrazioni allo schema rimico. Tuttavia molte tracce foneticamente, morfologicamente e lessicalmente riconducibili alla scripta aquitana, e dunque pittavino-guascona, in Marcabru e in Guglielmo IX, vengono corretti ed emendati dai canzonieri e relegati in sporadiche tracce della varia lectio. Già da qui possiamo notare come i canzonieri provenzali tendano, soprattutto quelli esemplati in area italiana, a per così dire «ripulire» $\mathrm{i}$ testi trobadorici dai tratti occitani non provenzali. Interessante è poi notare che alcuni tratti che Raimon Vidal stigmatizzava in Bernart de Ventadorn, non in sede di rima, non si trovino più nella varia lectio della tradizione manoscritta del trovatore, essendo perciò stati corretti dai copisti nei canzonieri. È il caso di amis per amics o chastiu per chastic nei canzonieri si trova sempre amics e chastic. Ciò vuol dire che tale discontinuità linguistica, tale rifondazione della lingua trobadorica, vinse. Probabilmente a tale azione rifondante delle prime grammatiche provenzali del XIII secolo è connessa anche la nascita della nuova forma di trasmissione del testo manoscritto, appunto il canzoniere. Ma questa è un'altra storia, di cui non è luogo trattare ora. 


\section{Bibliografia}

\section{Testi}

Avalle, D’A.S. (1962), Cultura e lingua francese delle origini nella «Passion» di Clermont-Ferrand, MilanoNapoli, Ricciardi.

Avalle, D’A.S. (1965), Sponsus. Dramma delle vergini prudenti e delle vergini stolte, Milano-Napoli, Ricciardi

Dejeanne, J.-M.-L. (1909), Poésies complètes du trobadour Marcabru, Toulouse, Privat.

Gaunt, S. - Harvey, R. - Paterson, L. (2000) Marcabru. A Critical Edition, Cambridge, D.S. Brewer.

Lazzerini, L. (1990), «Marcabru, A l'alena del vent doussa (BdT 293,2): proposte testuale o intepretative», in Messana, 4, pp. 47-87.

Lazzerini, L. (1992), «Un caso esemplare: Marcabru IV, Al prim comens de l'ivernaill», in Medioevo romanzo, $17, \mathrm{pp} .7-42$.

Ricketts, P.T. (1968), «Al'alena delvent doussa de Marcabru: édition critique, traduction et commentaire», in Revue des Langues romanes, 78, pp. 109-115.

Roncaglia, Au. (1953), «Marcabruno: Al departir del brau tempier [BdT 293,3]», in Cultura neolatina, 13, pp. 5-33.

Schwarze, Ch. (1963), Der Altprovenzalische Boeci, Münster, Verlag Aschendorff.

Viel, R. (2011), Troubadours mineurs gascons du XII siècle. Alegret, Marcoat, Amanieu de la Broqueira, Peire de Valeria, Gausbert Amiel. Édition critique bilingue avec introduction, notes et glossaire par Riccardo Viel, Paris, Champion.

\section{Studî}

Barachini, G., Viel, R. (2016) «Valore lessicale della suffissazione -uc nel sistema rimico dei trovatori», in Coluccia R., Brincat, J.M. - Möhren, F. (a cura di), Actes du XXVIIe Congrès international de linguistique et de philologie romanes (Nancy, 15-20 juillet 2013). Section 5: Lexicologie, phraséologie, lexicographie, Nancy, ATILF, pp. 37-48.

Beltrami, P. G. - Vatteroni S. (1988-94), Rimario trobadorico provenzale, 2 voll., Firenze, Pacini.

Bouvier, J.-C. (1976) Les parlers provençaux de la Drôme. Étude de géographie phonétique, Paris, Klincksieck.

FEW, Wartburg, W. von (1922-), Franæösisches Etymologisches Wörterbuch, 25 voll., Bonn/Leipzig/ Paris/Basel, Zbinden/Champion. 
Grafström, A. (1968), Étude sur la morphologie des plus anciennes chartes languedociennes, Stokholm, Almqvist \& Wiskell.

Guessard, F. (1973 [1a ed. 1858]) Grammaire provençales de Hugue Faidit et de Raymond Vidal de Besaudun (XIIle siècle). Deuxième édition revue, corrigée et considérablement augmentée par F. Guessard, Genève, Slatkine Reprints.

Jensen, F. (1986) «Deviations from the Troubadour Norm in the Language of Guillaume IX», in Studia occitanica in memoriam Paul Remy, a cura di Hans-Erich Keller, Kalamazoo, Michigan, II, pp. 347-362.

LRL (2001-2005), Lexicon der Romanistischen Linguistik, a cura di Günter Holtus, Michael Metzeltin, Christian Schmitt, Tübingen, Max Niemeyer, 12 voll.

M. Perugi, «Per un'analisi stratigrafica delle poesie di Marcabruno. Note in margine a una nuova edizione critica», in Studi Medievali, 44 (2003), pp. 533-600.

Meneghetti, M. L. (1993), «Una serrana per Marcabru?», in O cantar dos trobadores. Actas do Congreso celebrado en Santiago de Compostela entre os días 26 e 29 de abril de 1993, Xunta de Galicia, Santiago de Compostela, pp. 187-198.

Palay, S. (1961) Dictionnaire du béarnais et du gascon modernes (Bassin Aquitain) embrassant les dialectes du Béarn, de la Bigorre, du Gers, des Landes et de la Gascogne Maritime et Garonnaise, Paris, Éditions du Centre national de la recherche scientifique.

Pfister, M. (1976) «La langue de Guilhelm IX, comte de Poitiers», in Cahiers de Civilisation Médiévale, XIX, pp. 91-113.

Pignon, J. (1960) L'évolution phonétique des parlers du Poitou (Vienne et Deux-Sèvres), Paris, Édition d'Artrey.

Rohlfs, G. (1970), Le gascon. Études de philologie pyrénéenne, Tübingen, Max Niemeyer.

Ronjat, J. (1932), Grammaire istorique des parlares provençaux modernes, Montpellier, Société des Langues Romanes.

Viel, R. (2014) «Dopo l'edizione critica dei trovatori minori guasconi: nodi storici, linguistici ed ecdoticis, in Cultura neolatina, LXXIV/1-4, pp. 283-307.

Viel, R. (2015), «Interferenze linguistiche e tradizione manoscritta: alcune note su Marcabru», in Critica del testo, XVIII/3, pp. 3-27.

Viel, R. (2017), «Sulle tracce di una fonte antica: la diffusione dei primi trovatori», in I confini della lirica. Tempi, luoghi, tradizione della poesia romanza, a cura di Decaria A., Lagomarsini C., Firenze, Edizioni del Galluzzo, pp. 115-132. 